\title{
Computational Argumentation: \\ A Journey Beyond Semantics, Logic, Opinions, and Easy Tasks Invited talk
}

\author{
Ivan Habernal \\ UKP Lab, Technische Universität Darmstadt \\ habernal@ukp. informatik.tu-darmstadt.de
}

\begin{abstract}
The classical view on argumentation, such that arguments are logical structures consisting of different distinguishable parts and that parties exchange arguments in a rational way, is prevalent in textbooks but nonexistent in the real world. Instead, argumentation is a multifaceted communication tool built upon humans' capabilities to easily use common sense, emotions, and social context. As humans, we are pretty good at it. Computational Argumentation tries to tackle these phenomena but has a long and not so easy way to go. In this talk, I would like to shed a light on several recent attempts to deal with argumentation computationally, such as addressing argument quality, understanding argument reasoning, dealing with fallacies, and how should we never ever argue online.
\end{abstract}

